\title{
Análise de parâmetros perceptivo-auditivos e acústicos em indivíduos gagos
}

\section{Analysis of acoustic and auditory-perceptual parameters in stutterers}

\author{
Bruna Ferreira Valenzuela de Oliveira ${ }^{1}$, Erika Queiroga Werkhaizer Soares ${ }^{2}$, Luciana Lemos de Azevedo \\ Denise Brandão de Oliveira e Britto ${ }^{4}$
}

\begin{abstract}
RESUMO
Objetivo: Analisar parâmetros perceptivo-auditivos e acústicos da voz em indivíduos adultos gagos. Métodos: Foram analisados 15 indivíduos gagos do gênero masculino na faixa etária de 21 a 41 anos (média 26,6 anos), atendidos no Centro Clínico de Fonoaudiologia da instituição no período de fevereiro de 2005 a julho de 2007. Os parâmetros perceptivo-auditivos analisados envolveram a qualidade vocal, tipo de voz, ressonância, tensão vocal, velocidade de fala, coordenação pneumofônica, ataque vocal e gama tonal; quanto aos parâmetros acústicos, foram analisadas a frequência fundamental e sua variabilidade durante a fala espontânea. Resultados: A análise perceptivo-auditiva mostrou que as características mais frequentes nos indivíduos gagos foram: qualidade vocal normal (60\%), ressonância alterada (66\%), tensão vocal (86\%), ataque vocal alterado (73\%), velocidade de fala normal (54\%), gama tonal alterada (80\%) e coordenação pneumofônica alterada (100\%). No entanto, a análise estatística revelou que apenas a presença de tensão vocal, coordenação pneumofônica e a gama tonal alteradas apresentaram-se estatisticamente significativas nos indivíduos gagos estudados. Na análise acústica, a frequência fundamental variou de 125,54 a 149,59 Hz e a variabilidade da frequência fundamental foi de 16 a 21 semitons ou 112,50 a 172,40 Hz. Conclusão: Os parâmetros perceptivo-auditivos analisados que tiveram frequência significativa nos indivíduos gagos estudados foram: presença de tensão vocal, alteração da gama tonal e na coordenação pneumofônica. Desta forma, é importante avaliar os aspectos vocais nesses pacientes, pois a desordem da fluência pode comprometer alguns parâmetros vocais podendo ocasionar disfonia.
\end{abstract}

Descritores: Voz; Gagueira; Respiração; Fonação; Acústica da fala; Percepção auditiva

\section{INTRODUÇÃO}

A gagueira é um distúrbio da fluência caracterizado por interrupções anormais no fluxo de fala que ocorrem de forma involuntária ${ }^{(1-3)}$. Há diversas teorias que explicam a causa da gagueira, no entanto, até hoje não se estabeleceu sua etiologia. Acredita-se que a gagueira esteja relacionada a anormalidades hereditárias no funcionamento cerebral; especula-se também

Trabalho realizado na Pontifícia Universidade Católica de Minas Gerais PUC - Belo Horizonte (MG), Brasil.

(1) Pós-graduanda (Mestrado) do Programa de Pós-graduação em Estudos Linguísticos da Universidade Federal de Minas Gerais - UFMG - Belo Horizonte (MG), Brasil.

(2) Mestre, Professora da Pontifícia Universidade Católica de Minas Gerais - PUC - Belo Horizonte (MG), Brasil.

(3) Doutora, Professora da Pontifícia Universidade Católica de Minas Gerais - PUC - Belo Horizonte (MG), Brasil.

(4) Pós-graduanda (Doutorado) em Linguística e Língua Portuguesa do Programa de Pós-graduação em Letras da Pontifícia Universidade Católica de Minas Gerais - PUC - Belo Horizonte (MG), Brasil; Professora da Pontifícia Universidade Católica de Minas Gerais - PUC - Belo Horizonte (MG), Brasil. Endereço para correspondência: Bruna Ferreira Valenzuela de Oliveira. R. Cássia, 332/102, Bairro Prado, Belo Horizonte (MG), Brasil, CEP 30410-410. E-mail: brunafvo@yahoo.com.br

Recebido em: 8/4/2008; Aceito em: 9/10/2008 sobre fatores genéticos relacionados à predisposição para gaguejar, herança biológica e fatores hormonais; aceita-se ainda que influências ambientais e características de personalidade interfiram na gagueira. Além disso, fatores emocionais e o contexto social parecem contribuir para a sua manutenção. Atualmente, vários autores sustentam a idéia da etiologia múltipla para a gagueira( ${ }^{(4-5)}$.

Os sintomas da gagueira são classificados em primários e secundários. Os sintomas primários referem-se àqueles que podem ser observados pelo ouvinte, e incluem as pausas, bloqueios, revisões, interjeições, repetições e prolongamentos de um som, sílaba ou palavra monossilábica. Os sintomas secundários estão relacionados aos primários, mas não diretamente à fala, e são considerados reações utilizadas para evitar que se perceba a inabilidade na fala; dentre esses estão incluídos o aumento da tensão física; emoções negativas associadas à fala como medo e vergonha; contorções faciais; movimentos de braços, cabeça ou mandíbula; tensão cervical e de cintura escapular; tremor nos lábios; uso do ar residual durante a fala e anormalidades vocais, caracterizadas como pequenos espasmos na laringe acompanhados de "voz rachada" no momento da articulação ${ }^{(6-8)}$.

A prosódia também se encontra prejudicada. As pausas, 
repetições e bloqueios fazem com que os gagos tenham dificuldade em executar as mudanças necessárias no trato vocal para conseguir um acento melódico adequado à fluência normal. Os gagos apresentam dificuldade no planejamento dos traços supra-segmentais dos fonemas, o que dificulta o planejamento dos gestos articulatórios. Desta forma, indivíduos disfluentes apresentam variabilidade de frequência fundamental $\left(\mathrm{F}_{0}\right)$ e extensão de frequências mais restritas ${ }^{(9)}$.

A gagueira pode ser acompanhada por extrema tensão. Os bloqueios com voz podem ocorrer nos lábios, na ponta da língua contra o palato e nas pregas vocais; podem ainda ocorrer bloqueios sem voz, em todos os níveis, e a produção de fala durante a inspiração ${ }^{(10)}$. Essa característica de tensão, frequente na gagueira, é atribuída ao fato do indivíduo antecipar a gagueira na fala ainda não emitida como forma de evitá-la; no entanto, esse mecanismo produz mais tensão, ocasionando mais rupturas na fala. As emoções e os sentimentos de medo e ansiedade também aumentam a tensão durante a fala ${ }^{(11)}$. $\mathrm{O}$ esforço corporal é percebido nitidamente pelas contrações corporais exageradas, muitas vezes por gestos corporais indevidos ou por seu excesso ou pouca naturalidade ${ }^{(9)}$.

A quantidade excessiva de bloqueios e o esforço durante a fala são características frequentes em indivíduos gagos. Diante dessa consideração, passou-se a acreditar na possibilidade de uma causa física para a gagueira. A partir da observação de crianças gagas, verificou-se que havia uma expansão das narinas antes do bloqueio ocorrer. Essa expansão das narinas parecia abrir a passagem respiratória de forma a facilitar a entrada do ar. Esse fenômeno foi chamado de reflexo da dilatação da via aérea. Foi observado também que as pregas vocais abriam-se previamente a cada situação de gagueira e isso ocorria juntamente com a dilatação das narinas. A partir disso, supôs-se que o gago, diante de uma situação de estresse ou urgência em falar, faria com que seu cérebro interpretasse mal a informação da pressão intra-oral, como se fosse resultado de uma contração. Desta forma, o reflexo seria disparado, as pregas vocais se abririam largamente e o gago ficaria temporariamente sem fala. Esse fenômeno foi denominado laringoespasmo condicionado e se caracteriza por excessiva tensão e bloqueio das pregas vocais ${ }^{(12)}$.

Nos adultos, observa-se variedade de formas de bloqueios, envolvendo várias partes do corpo, não somente a face. Considerou-se a existência de um estresse de base que levaria ao aumento da tensão dos músculos do corpo em momentos desconfortáveis; quanto maior o estresse, maior seria o fechamento das pregas vocais ${ }^{(12)}$.

Alguns autores ${ }^{(13)}$ estudaram as desordens laríngeas encontradas na gagueira devido à grande frequência de bloqueios de sonoridade, distúrbios de melodia, alterações do ritmo pneumofônico, inspiração e expiração prolongadas, interrupção total da respiração, tentativas de falar durante a inspiração, alterações de intensidade vocal, tensão cervical e alterações tônico-posturais, que são observados em suas práticas clínicas. Como resultados, foi possível verificarem a existência de alterações funcionais laríngeas na gagueira, caracterizadas por maior lentidão na preparação das pregas vocais para fonação, além de perturbações no controle adutor e abdutor da laringe. Além disso, a adução glótica foi associada aos prolongamentos de sons, enquanto a abdução ocorre mais frequentemente durante as repetições e rupturas na fala. Não houve concordância quanto a hiperatividade da musculatura intrínseca da laringe ${ }^{(13)}$.

Alguns estudos ${ }^{(14-15)}$ foram realizados com o objetivo de descrever objetivamente o comportamento da laringe dos gagos durante momentos de gagueira na conversação. Um deles analisou 9 indivíduos por meio do exame de fibronasolaringoscopia, sendo possível sugerir que o comportamento laríngeo dos gagos difere entre os vários tipos de disfluências e que as características vocais dos sons podem ser influenciadas por este comportamento. Como resultado, foi observado que a maioria $(57 \%)$ dos prolongamentos de sons estava associada à adução glótica, enquanto para as repetições de sons ou sílabas foram observados quantidade aproximadamente equivalente de comportamento adutor (31\%) e abdutor (30\%). Com relação à sonoridade do som, os prolongamentos de sons vozeados em início de palavra estavam associados ao comportamento adutor $(72 \%)$, enquanto as repetições de sons e sílabas vozeadas estavam associadas ao comportamento abdutor ou intermediário da laringe, uma vez que apenas em $42 \%$ foi observado comportamento adutor. Quanto ao prolongamento de sons desvozeados também foi observado mais frequentemente (44\%) o comportamento aduzido. Já para as repetições de sons e sílabas desvozeadas não houve prevalência quanto ao comportamento adutor ou abdutor ${ }^{(14)}$.

Um outro estudo investigou a atividade da musculatura laríngea durante o discurso fluente e disfluente de quatro indivíduos do sexo masculino, por meio da eletromiografia. Os resultados sugeriram a existência de um componente laríngeo na gagueira, mostrando uma forte relação entre a atividade anormal da musculatura laríngea e os momentos de gagueira, devido aos níveis elevados de atividade muscular e ao rompimento da reciprocidade normal entre a adução e abdução dos grupos musculares ${ }^{(15)}$.

É importante ressaltar que a literatura carece de estudos recentes sobre o comportamento da laringe em indivíduos gagos. No entanto, a partir destes estudos citados sugere-se que a gagueira pode ocasionar modificações no funcionamento da laringe, expresso principalmente pelo aumento da atividade muscular relacionado à tensão. Entretanto, esse comportamento laríngeo não é considerado etiologia da gagueira, mas sim consequência das rupturas na fala.

Um estudo mais recente foi realizado através da eletromiografia com o objetivo de comparar a atividade muscular em indivíduos fluentes e gagos durante tarefas de fala e nãofala. A atividade muscular foi captada por eletrodos fixados no músculo orbicular inferior, musculatura suprahióidea, músculo esternocleidomastóideo e porção média do trapézio. Como resultados, não houve diferença na tensão de repouso entre gagos e fluentes; além disso, os gagos apresentaram tempo de reação de fala mais longo; atividade muscular durante a tarefa não verbal semelhante à observada durante o repouso e a atividade muscular dos gagos e fluentes durante a tarefa verbal foi similar. Os resultados sugerem que os gagos apresentam um pobre controle temporal para a coordenação dos processos motores ${ }^{(16)}$. É importante ressaltar que, além da função de avaliação, a eletromiografia tem sido utilizada 
como monitoramento no tratamento da gagueira, apresentando resultados satisfatórios na redução da mesma ${ }^{(17)}$.

Outro estudo investigou se falantes gagos e fluentes diferem na coordenação dos movimentos da boca e da laringe durante a produção de fala fluente. Para tanto, foi avaliada a produção da oclusiva bilabial desvozeada/p/, mas os grupos mostraram padrões semelhantes de tempo para os movimentos envolvidos na boca e laringe ${ }^{(18)}$.

Uma pesquisa realizada com o objetivo de medir a duração de consoantes na fala fluente de 20 informantes gagos, através da análise acústica, encontrou que a duração das consoantes produzidas por falantes gagos é significativamente menor do que as produzidas por falantes fluentes. Esses resultados indicaram uma velocidade de fala mais lenta para os falantes fluentes ${ }^{(19)}$. Em contrapartida, outro estudo realizado com o objetivo de caracterizar os aspectos da duração da emissão de fonemas plosivos e de logatomos do português brasileiro em indivíduos gagos e não gagos, encontrou que os indivíduos gagos, mesmo na fala fluente, apresentaram maior duração na produção dos fonemas e logatomos em relação ao grupo controle $^{(20)}$

Apesar da divergência dos estudos citados em relação à velocidade de fala, um estudo desenvolvido com 70 adultos gagos com o objetivo de relacionar a severidade da gagueira com a velocidade de fala, encontrou que quanto mais grave a gagueira, menor a velocidade de fala em palavras e sílabas por minuto. Tal achado sugere que a velocidade de fala deve ser incorporada às avaliações e tratamentos para gagueira ${ }^{(21)}$.

Em estudo realizado com o objetivo de verificar as alterações na estrutura prosódica em crianças gagas decorrentes das disfluências apresentadas pelas mesmas, concluiu-se que há uma importante relação entre disfluência e estrutura prosódica. Sendo assim, as características de fala do gago percebidas como fala monótona, lenta ou sem ritmo, podem estar relacionadas a esta estrutura prosódica particular ${ }^{(22)}$.

Como referido anteriormente, a gagueira envolve alguns sintomas relacionados à produção vocal. As contorções faciais e tensão cervical e de cintura escapular podem resultar em uma produção vocal tensa e com esforço. O uso do ar residual na fala pode provocar fadiga e redução na resistência vocal, alterando a qualidade vocal ${ }^{(23)}$. Dentre as anormalidades vocais citadas anteriormente, incluem-se também a alteração na intensidade e tonalidade da $\mathrm{voz}^{(6)}$, que estão diretamente relacionadas à produção vocal. Diante dessas alterações observadas em indivíduos gagos, nesse estudo será realizada a avaliação de alguns parâmetros perceptivo-auditivos da voz desses indivíduos, os quais serão descritos a seguir.

A avaliação perceptivo-auditiva é a avaliação clássica da qualidade vocal, muito frequente na prática clínica. A qualidade vocal se refere ao conjunto de características que identificam uma voz e está relacionada à impressão criada pela voz. O tipo de voz de um indivíduo se relaciona à seleção dos ajustes motores empregados, tanto nas pregas vocais e laringe, como em relação ao sistema de ressonância. Alguns tipos de vozes são encontrados mais frequentemente na prática clínica, dentre eles a voz rouca, com uma qualidade ruidosa e que indica irregularidade na vibração das pregas vocais; a voz áspera, que apresenta característica rude e desagradável, indicando rigidez na mucosa das pregas vocais; e a voz soprosa, que é acompanhada por ar não-sonorizado, tendo sido percebido ruído à fonação, indicando fluxo de ar não sonorizado através da glote ${ }^{(23)}$.

O sistema de ressonância vocal consiste em um conjunto de elementos do aparelho fonador que se relacionam entre si moldando e projetando o som no espaço. Envolve a amplificação da intensidade de sons de determinadas frequências da voz e o amortecimento de outras. O ideal é que se tenha uma ressonância equilibrada, onde o falante faz uso equilibrado desse sistema, sem concentrar a energia em nenhuma região específica do aparelho fonador. Sendo assim, a ressonância pode ser classificada como equilibrada, laríngea, faríngea, laringofaríngea, hiper ou hiponasal. Na ressonância laríngea, há uso excessivo da laringe, o que reflete em uma emissão tensa, com foco de ressonância baixo e sem projeção adequada. A ressonância faríngea também se caracteriza por tensão; no entanto, o foco de ressonância não é tão baixo quanto na laríngea e geralmente vem acompanhada de voz metálica e estridente. Quando há tensão da laringe e faringe, temos a ressonância laringofaríngea, caracterizada por voz comprimida, gerando um som abafado e sem projeção. Na ressonância hipernasal há uso excessivo da cavidade nasal, enquanto na hiponasal o uso da cavidade nasal é insuficiente ${ }^{(23)}$. Pelo fato dos indivíduos gagos apresentarem tensão excessiva durante a fala, é provável que eles apresentem ressonância laríngea ou faríngea, ou mesmo a associação de ambas.

$\mathrm{O}$ ataque vocal é a maneira como o som é iniciado pelo falante e se relaciona à configuração da glote no momento da emissão. Ele pode ser classificado como isocrônico, brusco ou soproso. No ataque vocal brusco ocorre uma forte adução das pregas vocais em toda sua extensão; já o ataque vocal soproso ocorre quando a expiração antecede a vibração das pregas vocais. O ideal é que o falante utilize o ataque vocal isocrônico na maior parte do tempo, onde a expiração coincide com o início da vibração da mucosa das pregas vocais; desta forma, não há perda de ar ou excesso de tensão ${ }^{(23)}$. Assim como a tensão excessiva à fonação e os bloqueios podem prejudicar a ressonância, é provável também que os indivíduos gagos apresentem ataque vocal brusco na maior parte de suas emissões.

A coordenação pneumofonoarticulatória (CPFA) resulta da inter-relação harmônica das forças expiratórias, mioelásticas da laringe e musculares da articulação. Quando essa coordenação não ocorre harmonicamente observa-se o predomínio de um desses níveis. A incoordenação pneumofônica pode comprometer a inteligibilidade de fala ${ }^{(23)}$, como ocorre muitas vezes com indivíduos gagos. Isso pode acontecer em decorrência da incoordenação pneumofônica devido ao predomínio das forças mioelásticas da laringe. É importante ressaltar que essa inteligibilidade de fala comprometida também está relacionada à velocidade de fala geralmente aumentada destes pacientes.

A gama tonal se refere à variação da frequência fundamental durante a fala encadeada. Pode ser classificada em normal, monoaltura, excessiva ou repetitiva. A monoaltura se caracteriza por fala monótona e restrita, com pouca variação na prosódia; já a gama tonal excessiva é caracterizada por variações extremas. A repetitiva caracteriza-se pelo mesmo padrão de entonação ${ }^{(23)}$. A fala dos indivíduos gagos, na maioria 
das vezes, soa como monótona ao ouvinte em decorrência da restrição na variação prosódica. Desta forma, é provável que a maioria dos gagos apresente gama tonal classificada como monoaltura ou repetitiva.

Os estudos na área de voz que abordam análise perceptivoauditiva geralmente são realizados com avaliação por cerca de três a sete fonoaudiólogos com especialização em voz ${ }^{(24-25)}$. Em relação ao tempo de gravação, apesar de poucos estudos analisarem fala espontânea, encontra-se amostras de fala variando de 39 segundos a dois minutos ${ }^{(25)}$, as quais são ouvidas geralmente por três vezes ${ }^{(26-27)}$.

$\mathrm{A}_{\mathrm{F}_{0}}$ é um parâmetro acústico relacionado à frequência da vibração das pregas vocais e reflete suas características biodinâmicas e sua integração com a pressão subglótica ${ }^{(23)}$. Seus valores variam em função do gênero e da idade, sendo que nas vozes masculinas a $\mathrm{F}_{0}$ pode variar de 80 a $150 \mathrm{~Hz}$, enquanto no sexo feminino os valores estão entre 150 e 250 Hz. No caso de crianças encontram-se valores acima de 250 $\mathrm{Hz}$. Outro parâmetro relacionado à $\mathrm{F}_{0}$ é a sua variabilidade, a qual deve ocorrer durante a fala; porém, variabilidade demais ou de menos é indesejável. Indivíduos disfluentes apresentam variabilidade de $\mathrm{F}_{0}$ e extensão de frequências mais restritas ${ }^{(23)}$.

Na prática clínica observamos algumas características vocais frequentes em pacientes gagos. Dentre elas destacamos a tensão à emissão vocal durante a fala, velocidade de fala aumentada e incoordenação pneumofônica. Desta forma, surge a necessidade de se estudar as características vocais mais frequentes em indivíduos gagos, a fim de que, com o enfoque também nos aspectos vocais, a conduta terapêutica seja beneficiada.

O objetivo do presente estudo foi analisar parâmetros perceptivo-auditivos e acústicos da voz em indivíduos adultos gagos. Os parâmetros perceptivo-auditivos analisados neste estudo envolveram a qualidade vocal, tipo de voz, ressonância, tensão vocal, velocidade de fala, CPFA, ataque vocal e gama tonal. Quanto aos parâmetros acústicos, foram analisadas a $\mathrm{F}_{0}$ e sua variabilidade durante a fala espontânea.

\section{MÉTODOS}

O presente estudo foi aprovado pelo Comitê de Ética em Pesquisa da Pontifícia Universidade Católica de Minas Gerais (CAAE 0014.0.213.000-07). Todos os indivíduos assinaram Termo de Consentimento Livre e Esclarecido conforme procedimento adotado no Centro Clínico de Fonoaudiologia da instituição, no início do tratamento fonoaudiológico, autorizando a divulgação dos dados coletados durante o tratamento, em pesquisas científicas.

Foram realizadas análises perceptivo-auditiva e acústica da voz, em produção de fala espontânea, de 15 indivíduos gagos do gênero masculino na faixa etária de 21 a 41 anos (média 26,6 anos), atendidos no Centro Clínico de Fonoaudiologia da Pontifícia Universidade Católica de Minas Gerais (PUCMinas), no período de fevereiro de 2005 a julho de 2007. Os parâmetros perceptivo-auditivos analisados foram: qualidade vocal, tipo de voz, ressonância, tensão vocal, velocidade de fala, CPFA, ataque vocal e gama tonal. Quanto aos parâmetros acústicos foram analisadas a $\mathrm{F}_{0}$ e sua variabilidade.
Foram selecionados os indivíduos que apresentassem gagueira de acordo com a classificação da prova de fluência do teste de linguagem $\mathrm{ABFW}$, que apesar de ser um teste de linguagem infantil, utiliza, na prova de fluência, parâmetros de normalidade para adultos. Pelo fato da gagueira ser mais frequente no gênero masculino ${ }^{(5)} \mathrm{e}$, em nossa prática clínica também observarmos essa característica, optamos por selecionar apenas indivíduos deste gênero.

A amostra de fala espontânea foi coletada antes do início do tratamento fonoaudiológico (durante a avaliação) por meio de gravação digital realizada em computador Pentium IV, 1.8 GHz, com placa de som Via AC'97 Sound Blaster, em ambiente acusticamente tratado. Para a captação da voz foi utilizado microfone Shure BG 1.1 unidirecional devidamente conectado ao computador, posicionado lateralmente à boca do paciente a uma distância de cinco centímetros. O paciente foi orientado a dizer o motivo que o levou a procurar o tratamento fonoaudiológico, o que esperava do tratamento e as impressões que tinha sobre sua fala. O tempo de gravação foi de aproximadamente três minutos, que se refere ao tempo mínimo de coleta de amostra de fala espontânea exigido pelo protocolo de avaliação de fluência do Centro Clínico de Fonoaudiologia da PUC-Minas onde foi realizada a pesquisa, para que se possa realizar a análise com a amostra de 200 sílabas fluentes sugerida pela literatura especializada.

As gravações foram apresentadas em CD a cinco fonoaudiólogas especialistas em voz que possuíam no mínimo cinco anos de experiência na área, as quais realizaram a análise perceptivo-auditiva da voz dos indivíduos. Para análise da amostra de fala, as profissionais deveriam marcar seus achados em um protocolo (Anexo 1) que avalia a qualidade vocal, grau de alteração, o tipo de voz observado, ressonância, tensão vocal, velocidade de fala, CPFA, ataque vocal e gama tonal. Neste protocolo, as fonoaudiólogas foram solicitadas a marcar apenas uma opção de cada parâmetro avaliado, exceto para os parâmetros de ressonância e tipo de voz, onde foi permitida a marcação de mais de uma opção. Cada gravação foi apresentada às fonoaudiólogas três vezes. Primeiramente, elas fizeram uma leitura detalhada do protocolo com o objetivo de se familiarizarem com o mesmo e conhecerem o que seria observado nas amostras que lhes seriam apresentadas. Em seguida, foi apresentada a primeira amostra do CD, quando as fonoaudiólogas apenas escutaram a gravação. Logo após, a amostra foi reapresentada e as profissionais ouviram e marcaram no protocolo o seu parecer fonoaudiológico. E, finalmente, a amostra foi reapresentada para que as fonoaudiólogas confirmassem suas observações. $\mathrm{O}$ processo se repetiu para cada indivíduo analisado. Em estudo preliminar (piloto) constatamos que o número de repetições da amostra de fala não poderia ser inferior a três e não havia necessidade de ser maior.

A análise acústica foi realizada por meio do programa de análise acústica Praat 4.5.15 através do qual foram obtidos os valores de $\mathrm{F}_{0}$ (em Hz) e sua variabilidade (em Hz e semitons), os quais foram transcritos em protocolo específico (Anexo 2).

A análise estatística foi realizada no software Minitab versão 13.20. Com os dados da análise acústica foi realizada inferência estatística com a montagem de intervalos de con- 
fiança para a média de $\mathrm{F}_{0}$ e sua variabilidade. Já para a análise perceptivo-auditiva foram realizados além dos intervalos de confiança, testes de hipótese para a proporção de modo a testar a hipótese nula $\mathrm{H}_{0}$ de igualdade de proporções. Caso ocorresse valor $\mathrm{p}$ menor que o nível de significância determinado a priori, de $5 \%$, rejeitava-se a hipótese de igualdade de proporções para cada classificação dos parâmetros perceptivo-auditivos. Neste sentido, a hipótese alternativa $\mathrm{H}_{1}$ era validada, tornando possível considerar determinado parâmetro perceptivo-auditivo como característica significativamente frequente nos indivíduos estudados.

\section{RESULTADOS}

Após análise perceptivo-auditiva da fala espontânea realizada pelas cinco fonoaudiólogas, não se encontrou ocorrência significativa de respostas dentro de cada parâmetro analisado, impossibilitando a caracterização de um achado mais frequente. Assim, os parâmetros em que havia mais de uma opção (ressonância, ataque vocal, velocidade de fala e gama tonal), foram agrupados de forma a considerar as respostas apenas como "normal" ou "alterado". Desta forma, a ressonância foi classificada como equilibrada ou alterada (o que incluía os subtipos: hipernasal, hiponasal, laríngea, faríngea); o ataque vocal em isocrônico ou alterado (brusco ou aspirado); a velocidade de fala em adequada ou alterada (quando classificada como: reduzida, aumentada ou muito variada) e a gama tonal em normal ou alterada (restrita, repetitiva, monoaltura ou excessiva).

Em relação à qualidade vocal, a maioria da amostra, 9 (60\%) foi classificada como normal. Nos 6 pacientes (40\%) que apresentaram qualidade vocal alterada, o grau de alteração leve esteve presente em todos. $\mathrm{O}$ tipo de voz rouca foi o mais frequente, estando presente em 4 (66\%) sujeitos; além disso, os tipos de voz soprosa, crepitante e feminilizada estiveram presentes em 1 (16\%) indivíduo, cada um, conforme ilustrado na Figura 1.

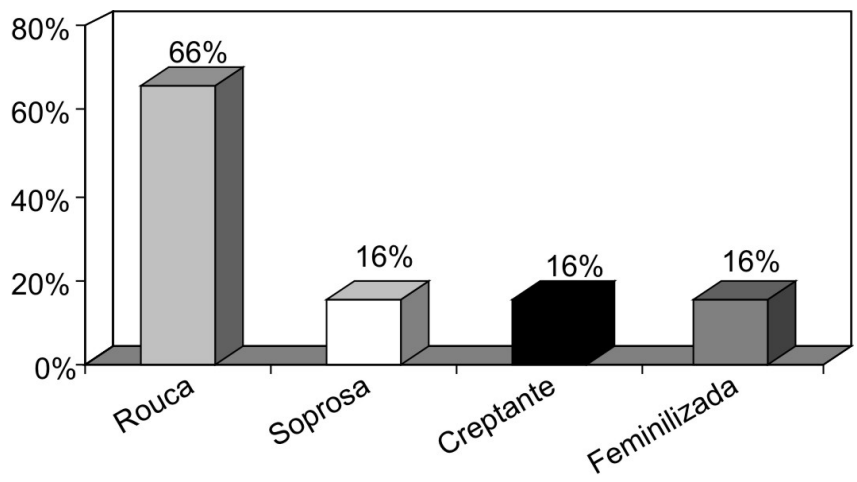

Figura 1. Porcentagem de tipos de voz dos indivíduos gagos com qualidade vocal alterada

O estudo do intervalo de confiança aponta que a proporção de gagos estudados que possuem qualidade vocal normal está entre $32,28 \%$ e $83,66 \%$. O valor de p encontrado para este parâmetro $(\mathrm{p}=0,607)$ não foi estatisticamente significativo, uma vez que foi maior do que 0,05 .
A ressonância esteve alterada em $10(66 \%)$ pacientes e a tensão vocal presente em 13 (86\%) indivíduos da amostra. Em relação ao ataque vocal, 11 (73\%) apresentaram ataque vocal alterado, podendo ser brusco ou aspirado. A CPFA foi percebida como alterada nos $15(100 \%)$ pacientes da amostra.

Quanto à velocidade de fala, 7 (46\%) apresentaram alteração e, em relação à gama tonal $12(80 \%)$ sujeitos possuíam alteração deste parâmetro.

A análise estatística de cada parâmetro perceptivoauditivo foi realizada baseando-se na classificação mais frequente de cada parâmetro. Desta forma, o teste de hipótese foi realizado para as seguintes classificações: qualidade vocal normal, ressonância alterada, tensão vocal presente, ataque vocal alterado, CPFA alterada, velocidade de fala alterada e gama tonal alterada; as quais foram estabelecidas como hipóteses alternativas para o teste de hipótese. Pelo fato da qualidade vocal normal ter sido mais frequente, não foi realizada análise estatística para o grau de alteração, bem como para tipo de voz, sendo realizada então, apenas análise descritiva da amostra.

Assim como a qualidade vocal ( $\mathrm{p}=0,607)$, os parâmetros de ressonância $(\mathrm{p}=0,151)$, ataque vocal $(\mathrm{p}=0,059)$ e velocidade de fala $(\mathrm{p}=0,696)$ apresentaram valor $\mathrm{p}$ maior que $5 \%$. Desta forma, as classificações mais frequentes desses parâmetros não foram vistas com frequência significativa nos indivíduos gagos estudados.

Em contrapartida, os parâmetros de tensão vocal $(\mathrm{p}=0,004)$, CPFA $(p=0,000)$ e gama tonal $(p=0,018)$ apresentaram valores estatisticamente significativos. Desta forma, a presença de tensão vocal e alteração na CPFA e na gama tonal foram vistas com frequência significativa nos indivíduos estudados. Os valores p, bem como o intervalo de confiança para cada parâmetro perceptivo-auditivo analisado estão descritos na Tabela 1.

Tabela 1. Resultados de inferência para os dados amostrais em relação aos parâmetros perceptivo- auditivos

\begin{tabular}{lcc}
\hline $\begin{array}{l}\text { Parâmetros } \\
\text { perceptivo-auditivos }\end{array}$ & \multicolumn{1}{c}{$\begin{array}{c}\text { Intervalo de } \\
\text { confiança }\end{array}$} & $\begin{array}{c}\text { Valor } \\
\text { de } p\end{array}$ \\
\hline Qualidade vocal normal & $(0,322870 ; 0,836636)$ & 0,607 \\
Ressonância alterada & $(0,383804 ; 0,881759)$ & 0,151 \\
Tensão vocal presente & $(0,595397 ; 0,983424)$ & $0,004^{*}$ \\
Ataque vocal alterado & $(0,448997 ; 0,922128)$ & 0,059 \\
CPFA alterada & $(0,818964 ; 1,000000)$ & $0,000^{*}$ \\
Velocidade de fala alterada & $(0,212667 ; 0,734139)$ & 0,696 \\
Gama tonal alterada & $(0,519109 ; 0,956688)$ & $0,018^{*}$ \\
\hline Legenda: CPFA = coordenação pneumofonoarticulatória & \\
*Valores estatisticamente significativos & \\
Teste de hipótese para proporção $\left(\mathrm{H}_{0}: \mathrm{p}=0,5\right)$ e estimação por intervalo
\end{tabular}

Em relação à análise acústica, a $\mathrm{F}_{0}$ dos indivíduos gagos estudados variou de 125,54 a 149,59 Hz. A variabilidade da $\mathrm{F}_{0}$ em $\mathrm{Hz}$, por sua vez, variou de 112,50 a 172,40 Hz, enquanto em semitons a variação foi de 16 a 21, considerando mesmo coeficiente de confiança. Os valores de média, mediana, desvio padrão, além dos valores máximos e mínimos dos parâmetros acústicos analisados estão descritos na Tabela 2. 
Tabela 2. Estatística descritiva para os dados amostrais com relação aos parâmetros acústicos

\begin{tabular}{lccccc}
\hline Parâmetros acústicos & Média & Mediana & Desvio padrão & Valor mínimo & Valor máximo \\
\hline $\mathrm{F}_{0}$ & 137,56 & 141,97 & 21,72 & 101,83 & 164,99 \\
Variabilidade da $\mathrm{F}_{0}(\mathrm{~Hz})$ & 142,5 & 151,4 & 54,1 & 54,4 & 265,7 \\
Variabilidade da $\mathrm{F}_{0}$ (semitons) & 18,6 & 19 & 4,42 & 10 & 28 \\
\hline
\end{tabular}

Legenda: $F_{0}=$ frequência fundamental

Estimadores de tendência central e variabilidade

\section{DISCUSSÃO}

A partir da literatura, observa-se que os indivíduos gagos apresentam características de fala que podem contribuir para o desenvolvimento de alterações vocais.

A presença de rupturas na fala desses indivíduos pode ocasionar alteração na prosódia, uma vez que as mudanças no trato vocal necessárias para a produção de um acento melódico adequado são dificultadas ${ }^{(9)}$. Em crianças gagas, a estrutura prosódica também se encontra prejudicada, sendo caracterizada por fala monótona, lenta e sem ritmo ${ }^{(22)}$. Estes resultados coincidem os do presente estudo, uma vez que a gama tonal alterada foi vista com frequência significativa nos indivíduos estudados.

Dentre os $12(80 \%)$ indivíduos gagos que apresentaram gama tonal alterada, a mais frequente foi a repetitiva, $6(50 \%)$, seguida da restrita, 5 (42\%), e monoaltura 1 (8\%). Essa característica da gama tonal observada nos indivíduos estudados é refletida por uma restrição na variação da $\mathrm{F}_{0}$ durante a fala, o que compromete a melodia do discurso, deixando a fala monótona e sem modulação.

Em relação à análise acústica observamos que a $\mathrm{F}_{0}$ dos indivíduos gagos estudados variou de 125,54 a $149,59 \mathrm{~Hz}$, o que está de acordo com a literatura ${ }^{(23)}$, que considera que a $\mathrm{F}_{0}$ para indivíduos do sexo masculino varia de 80 a $150 \mathrm{~Hz}$. Desta forma, os gagos do gênero masculino não possuem $\mathrm{F}_{0}$ diferente da apresentada por indivíduos fluentes.

Quanto à variabilidade da $\mathrm{F}_{0}$ na fala espontânea, não há valores de normalidade na literatura que possam ser comparados com os achados deste estudo. Considera-se apenas que indivíduos disfluentes apresentam variabilidade de $\mathrm{F}_{0} \mathrm{e}$ extensão de frequências mais restritas ${ }^{(23)}$.

A literatura considera que o ritmo da fala dos indivíduos gagos encontra-se alterado devido às acelerações indevidas durante a fala ${ }^{(9)}$. Alguns estudos ${ }^{(19-20)}$ foram realizados por meio da análise acústica, visando medir a duração de fonemas produzidos por indivíduos gagos. Em um deles observou-se que a duração das consoantes produzidas por gagos é menor do que as produzidas por indivíduos fluentes ${ }^{(19)}$, enquanto no outro estudo, a duração na produção dos fonemas foi maior nos gagos, quando comparados com um grupo controle ${ }^{(20)}$. No presente estudo, a alteração na velocidade de fala não apresentou valor estatisticamente significativo $(p=0,696)$ nos indivíduos estudados, quando analisada perceptivamente. Tal achado não coincide com a literatura, que apesar de não demonstrar concordância sobre a velocidade de fala dos gagos, considera que seja alterada.
As características de tensão e esforço durante a fala são frequentes em indivíduos gagos ${ }^{(10)}$. Em um estudo realizado através da eletromiografia, visando investigar a atividade da musculatura laríngea na gagueira, os resultados sugeriram a presença de uma relação significativa entre a atividade anormal desta musculatura e os momentos de gagueira ${ }^{(15)}$. Os achados do estudo citado coincidem com nossa pesquisa, onde a tensão vocal esteve presente em 13 (86\%) indivíduos e apresentou-se estatisticamente significativa $(\mathrm{p}=0,004)$ nos indivíduos gagos estudados, quando analisada perceptivamente.

No presente estudo, a tensão vocal foi percebida pelas fonoaudiólogas que realizaram a avaliação perceptivo-auditiva, como um esforço vocal durante a fala. Acredita-se que essa tensão vocal seja resultado das contorções faciais e da tensão cervical e de cintura escapular, frequentemente observada nos indivíduos gagos.

A literatura não apresenta achados em relação à ressonância dos indivíduos gagos. Entretanto, pelo fato desses possuírem tensão excessiva durante a fala, esperávamos encontrar ressonância alterada, principalmente sendo classificada como laríngea, faríngea ou associação de ambas. No entanto, embora neste estudo a ressonância esteja alterada em 10 (66\%) indivíduos, não apresentou valor estatisticamente significativo $(\mathrm{p}=0,151)$.

Em relação ao ataque vocal, 11 (73\%) indivíduos apresentaram alteração, porém essa característica não apresentou valor estatisticamente significativo $(\mathrm{p}=0,059)$ nos indivíduos estudados considerando o nível de significância de 5\% determinado neste estudo. No entanto, o ataque vocal alterado apresenta-se clinicamente significativo* nos indivíduos gagos estudados. Embora não hajam achados na literatura que caracterizem o ataque vocal nos gagos, a tensão excessiva à fonação e os bloqueios poderiam contribuir para que o ataque vocal brusco (alterado) ocorresse na maior parte da emissão destes indivíduos.

A CPFA alterada apresentou valor estatisticamente significativo $(\mathrm{p}=0,000)$ nos indivíduos gagos. A CPFA foi considerada alterada pelas avaliadoras quando os indivíduos gagos utilizavam o ar de reserva durante a amostra de fala analisada. É importante ressaltar que a incoordenação pneumofônica pode comprometer a inteligibilidade de fala e contribuir para a hiperfunção laríngea ${ }^{(23)}$. No entanto, a literatura não apresenta estudos que descrevam a CPFA em indivíduos gagos.

É importante ressaltar que os achados deste estudo não podem ser generalizados, pois foram analisados apenas 15 indivíduos gagos do gênero masculino em uma amostra de fala de apenas três minutos. O ideal seria analisar a fala espontânea

*Considerando um nível de significância de $10 \%$, o ataque vocal alterado nos indivíduos gagos apresenta-se estatisticamente significativo 
de indivíduos gagos de ambos os gêneros, em uma amostra de fala maior, e que fosse utilizado um grupo controle para a comparação dos achados entre os grupos, visando maior fidedignidade do estudo.

\section{CONCLUSÃO}

Com este estudo pôde-se observar que a qualidade vocal normal, ressonância alterada, ataque vocal alterado e velocidade de fala alterada, apesar de frequentes, não se mostraram estatisticamente significativos nos indivíduos gagos estudados. Entretanto, observamos frequência significativa de tensão vocal, além de CPFA e gama tonal alteradas. Além disso, a $\mathrm{F}_{0}$ não apresentou alterações nos indivíduos gagos estudados.
Apesar de os achados deste estudo não poderem ser generalizados, pode-se sugerir que é importante avaliar os aspectos vocais nos pacientes gagos, pois a desordem da fluência pode provocar alterações em alguns parâmetros vocais podendo ocasionar disfonia.

Sugere-se a realização de estudos futuros com uma amostra maior e com grupo controle a fim de que se possa obter resultados mais fidedignos e que possam ser generalizados para toda a população de indivíduos gagos. Além disso, sugere-se a realização de estudo visando padronizar os valores de variabilidade da $\mathrm{F}_{0}$ na fala espontânea de indivíduos com gama tonal normal, a fim de que se estabeleçam valores de normalidade para este parâmetro, possibilitando a comparação com os resultados deste e de outros estudos.

\begin{abstract}
Purpose: To analyze auditory-perceptual and acoustic parameters of the voices of adult stutterers. Methods: Fifteen male stutterers in the age range from 21 to 41 years (mean 26.6 years), attended at the institution's Speech-Language Pathology Clinical Center in the period from February 2005 to July 2007, were analyzed. The auditory-perceptual parameters analyzed were vocal quality, type of voice, resonance, vocal tension, speech rate, pneumo-phonic coordination, vocal attack and pitch range; the acoustic parameters analyzed were fundamental frequency and its variability during spontaneous speech. Results: The auditory-perceptual analysis showed that the most frequent characteristics among the subjects were normal vocal quality (60\%), altered resonance $(66 \%)$, vocal tension (86\%), altered vocal attack (73\%), normal speech rate (54\%), altered pitch range (80\%) and altered pneumo-phonic coordination $(100 \%)$. However, only the presence of vocal tension and the altered pneumo-phonic coordination and pitch range were statistically significant in the stutterers studied. In the acoustic analysis, fundamental frequency varied from 125,54 to 149,59 $\mathrm{Hz}$, and the variability of the fundamental frequency ranged from 16 to 21 halftones, or from 112,50 to 172,40 Hz. Conclusion: The auditory-perceptual parameters that were significantly frequent among stutterers were: presence of vocal tension, altered pneumophonic coordination, and altered pitch range. Therefore, it is important to evaluate the vocal aspects of these patients, for the fluency disorders might undermine some vocals parameters, causing dysphonia.
\end{abstract}

Keywords: Voice; Stuttering; Respiration; Phonation; Speech acoustics; Auditory perception

\section{REFERÊNCIAS}

1. Perkins WH. The problem of definition; commentary on "stuttering". J Speech Hear Disord. 1983;48:246-9.

2. Cooper EB. Red herrings, dead horses, straw men, and blind alleys; escaping the stuttering conundrum. J Fluency Disord. 1993;18:375-87.

3. Prins D. Models for treatment efficacy studies of adult stutterers. J Fluency Disord. 1993;18:333-49.

4. Perkins WH, Kent RD, Curlee RF. A theory of neuropsycholinguistic function in stuttering. J Speech Hear Res. 1991;34(4):734-52. Review.

5. Felsenfeld S. Progress and need in the genetics of stuttering. J Fluency Disord. 1996;21(2):77-103.

6. Jakubovicz R. A gagueira: teoria e tratamento de adultos e crianças. 5a. ed. Rio de Janeiro: Revinter; 1997.

7. Barbosa LMG. Noções básicas sobre a gagueira: suas características, sua etiologia e as teorias sobre a sua natureza. In: Ribeiro IM. Conhecimentos essenciais para atender bem a pessoa com queixa de gagueira. São José dos Campos: Pulso Editorial; 2003. p.17-32.

8. Finn P, Cordes AK. Multicultural identification and treatment of stuttering: A continuing need for research. J Fluency Disord. 1997; 22(2):219-36.

9. Cupello R. Fluência e disfluências. In: Cupello R. Gagueira: uma visão neuropsicológica - avaliação e tratamento. Revinter: Rio de Janeiro; 2007. p.15-26
10. Irwin A. Gagueira: uma ajuda prática em qualquer idade. São Paulo: Martins Fontes; 1993.

11. Oliveira PS. Gagueira: a teoria na prática. Rev CEFAC. 1999;1(2):56-64.

12. Schwartz MF, Jakubovicz R, Mourão ACC, Silva TC. A gagueira resolvida de Martin F. Schwartz, Toronto, Heinemann, 1976. J Bras Fonoaudiol. 2005;5(22):336-42.

13. Chaves ALGL, Pinho SMR, Assencio-Ferreira VJ. Aspectos laríngeos e fonatórios presentes na gagueira. Rev CEFAC. 2001;3(2):161-4.

14. Conture EG, Schwartz HD, Brewer DW. Laryngeal behavior during stuttering: a further study. J Speech Hear Res. 1985;28(2):233-40.

15. Freeman FJ, Ushijima T. Laryngeal muscle activity during stuttering. J Speech Hear Res. 1978;21(3):538-62.

16. Andrade CRF, Sassi FC, Juste FS, Meira MIM. Atividades de fala e não-fala em gagueira: estudo preliminar. Pró-Fono. 2008;20(1):67-70.

17. Andrade CRF, Sassi FC, Juste FS, Ercolin B. Modelamento da fluência com o uso da eletromiografia de superfície: estudo piloto. Pró-Fono. 2008;20(2):129-32.

18. Max L, Gracco VL. Coordination of oral and laryngeal movements in the perceptually fluent speech of adults who stutter. J Speech Lang Hear Res. 2005;48(3):524-42.

19. Pereira MMB, Ferrante C, Cohen C, Carvalho GTC. Análise da duração de consoantes na fala fluente de gagos. Rev Soc Bras Fonoaudiol. $2003 ; 8(2): 14-8$. 
20. Colacicco FB, Arcuri CF, Osborn E, Schiefer AM. Caracterização dos aspectos de duração da fala de indivíduos fluentes e gagos. Rev Soc Bras Fonoaudiol. 2006;11(3):158-62.

21. Andrade CRF, Cervone LM, Sassi FC. Relationship between the stuttering severity index and speech rate. Sao Paulo Med J. 2003;121(2):81-4.

22. Soares EQW. Aspectos prosódicos da fala na gagueira infantil [dissertação]. Belo Horizonte: Faculdade de Letras da Universidade Federal de Minas Gerais; 2004.

23. Behlau M, Madazio G, Feijó D, Pontes P. Avaliação de voz. In: Behlau M, organizadora. Voz: o livro do especialista. Rio de Janeiro: Revinter; 2001. p. 85-176.
24. Figueredo DC, Souza PRF, Gonçalves MIR, Biase NG. Análise perceptivo-auditiva, acústica e laringológica da voz de adultos jovens fumantes e não-fumantes. Rev Bras Otorrinolaringol. 2003;69(6):791-9.

25. Mariano NC, Silva MAA, Cury RI, Sousa Neto OM. Autopercepção da voz em estudantes do curso de graduação em fonoaudiologia da FCMSCSP. Acta ORL. 2007;25(2):131-40.

26. Gasparini G, Diaféria G, Behlau M. Queixa vocal e análise perceptivoauditiva de pacientes com doença de Parkinson. Rev Ciênc Méd Biol = J Med Biol Sci. 2003;2(1):72-6.

27. Cukier S, Camargo Z. Abordagem da qualidade vocal em um falante com deficiência auditiva: aspectos acústicos relevantes do sinal de fala. Rev CEFAC. 2005;7(1):93-101.

Anexo 1. Protocolo de avaliação perceptivo-auditiva

Prontuário do paciente: Data da gravação:

Fonoaudiólogo:

1 - Qualidade vocal

normal

$\square$ alterada

2 - Grau de alteração

$\square$ leve

moderado

$\square$ severo

a extremo

3 - Tipo de voz

$\square$ rouca

$\square$ áspera

a soprosa

$\square$ fluida

crepitante

$\square$ outros:

\section{4 - Ressonância}

$\square$ equilibrada

$\checkmark$ hipernasal

$\square$ hiponasal

$\square$ faríngea

$\square$ laríngea
5 - Tensão vocal

$\square$ presente

$\square$ ausente

6 - Tipo de ataque vocal

brusco

aspirado

$\square$ isocrônico

7 - Coordenação pneumofonoarticulatória:

$\square$ adequada

$\square$ alterada

8 - Velocidade de fala

normal

$\square$ reduzida

$\square$ aumentada

$\square$ muito variada

9 - Gama tonal

$\square$ normal

$\square$ excessiva

monoaltura

$\square$ repetitiva

$\square$ restrita 
Anexo 2. Protocolo de análise acústica

\begin{tabular}{|c|c|c|c|c|}
\hline \multirow{2}{*}{ Prontuário do paciente } & \multirow{2}{*}{ Data da gravação } & \multirow{2}{*}{$\mathrm{F}_{0(\mathrm{~Hz})}$} & \multicolumn{2}{|c|}{ Variabilidade da $\mathrm{F}_{0}$} \\
\hline & & & $\mathrm{Hz}$ & Semitons \\
\hline & & & & \\
\hline & & & & \\
\hline & & & & \\
\hline & & & & \\
\hline & & & & \\
\hline & & & & \\
\hline & & & & \\
\hline & & & & \\
\hline & & & & \\
\hline & & & & \\
\hline & & & & \\
\hline & & & & \\
\hline & & & & \\
\hline & & & & \\
\hline
\end{tabular}

